\title{
Physical exercise in locally advanced pancreatic adenocarcinoma: "If I walk, I live. Although one can die of cancer, now I am living"
}

\author{
Giovanni Lo $\operatorname{Re}^{1}$, Silvia Magnaldi' ${ }^{2}$ Paolo Doretto ${ }^{3}$, Roberto Innocente ${ }^{4}$, Francesco Lo $\mathbf{R e}^{5}$ \\ ${ }^{1}$ Medical Oncology and Immune-related tumors, CRO IRCCS, Aviano 33081, Italy. \\ ${ }^{2}$ Radiology, AAS5 Pordenonese, Pordenone 33170, Italy. \\ ${ }^{3}$ Clinical pathology, AAS5 Pordenonese, Pordenone 33170, Italy. \\ ${ }^{4}$ Radiotherapy, Centro di Riferimento Oncologico (CRO), Aviano 33081, Italy. \\ ${ }^{5}$ Chemotherapy and Medical Toxicology, Study University, Milan 20129, Italy.
}

Correspondence to: Dr. Giovanni Lo Re, Medical Oncology and Immune-related tumors, CRO IRCCS, Aviano 33081, Italy. E-mail: giovanni.lore@cro.it

How to cite this article: Lo Re G, Magnaldi S, Doretto P, Innocente R, Lo Re F. Physical exercise in locally advanced pancreatic adenocarcinoma: "If I walk, I live. Although one can die of cancer, now I am living". J Cancer Metastasis Treat 2019;5:81.

http://dx.doi.org/10.20517/2394-4722.2019.30

Received: 30 Aug 2019 First Decision: 28 Oct 2019 Revised: 11 Nov 2019 Accepted: 17 Nov 2019 Published: 17 Dec 2019

Science Editor: Pravin D. Potdar Copy Editor: Cai-Hong Wang Production Editor: Jing Yu

\begin{abstract}
The most widely used chemotherapeutic combinations in locally advanced (LA) or metastatic pancreatic ductal adenocarcinoma (PDAC) are Nab-Paclitaxel-Gemcitabine (Nab-PCT-GEM) and Fluorouracil, Folinic acid, Oxaliplatin, and irinotecan (FOLFIRINOX). Chemo-resistance, typical of PDAC, appears to be due to the negative influence of stroma population cells, namely regulatory $T$ cells (Treg) and myeloid-derived suppressor cells, macrophages with inhibitory effects on the antitumor activity of the innate and adaptive immune systems, and resistance to cancer treatment. Among other factors that may influence immune surveillance, constant physical activity appears to reduce the risk of cancer-related mortality and cardiovascular risk. However, this does not seem to increase the survival of patients with PDAC. The exception is our young inoperable patient. For LA head PDAC, he was treated with seven cycles of Nab-PCT-GEM and RT 50 Gy/15 fractions combined to biweekly GEM and salvage FOLFIRINOX. The five-year surviving patient travelled $15,000 \mathrm{~km}$ on foot and continues inexorably his "walking therapy".
\end{abstract}

Keywords: Pancreatic adenocarcinoma, depression, physical activity, Nab-paclitaxel, fluorouracil, regulatory T cells, myeloid-derived suppressor cells

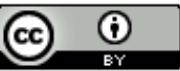

(C) The Author(s) 2019. Open Access This article is licensed under a Creative Commons Attribution 4.0 International License (https://creativecommons.org/licenses/by/4.0/), which permits unrestricted use, sharing, adaptation, distribution and reproduction in any medium or format, for any purpose, even commercially, as long as you give appropriate credit to the original author(s) and the source, provide a link to the Creative Commons license, and indicate if changes were made.

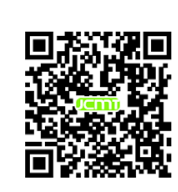




\section{INTRODUCTION}

The severity of pancreatic ductal adenocarcinoma (PDAC) is often linked to a late diagnosis with evidence of a locally advanced or metastatic disease, and the prognosis is poor. PDAC is one of the most chemoresistant tumors, which seems related to derived tumor-associated macrophages (TAMs) cells in the tumor's desmoplastic reaction ${ }^{[1]}$. They are distinguished in M1 macrophages, which express inducible nitric oxide synthase, major histocompatibility complex class II (MHC-II) proteins, and CD80, CD86, and tumor necrosis factor $\alpha$ proteins, and they have immunostimulatory activity. In contrast, M2 macrophages expressing Th2 cytokines, arginase 1, CD206, and low amounts of MHC-II have immunosuppressive functions that allow tumor progression ${ }^{[2]}$. Furthermore, the tumor microenvironment (TME), through $\operatorname{Treg}^{[3]}$ and myeloid-derived suppressor cells $(\mathrm{MDSC})^{[4]}$ cells, has negative immunosuppressive effects.

Current treatments for advanced PDAC that improve prognosis and survival are Nab-PaclitaxelGemcitabine (Nab-PCT-GEM) ${ }^{[5]}$ and FOLFIRINOX ${ }^{[6]}$. Furthermore, Nab-PCT-GEM regimen, in addition to its direct antitumor activity, seems to have immunoregulatory function. In fact, nab-PCT polarizes M1 macrophages by toll-like receptor 4 signaling ${ }^{[2,7]}$, whereas GEM has a dual action on MDSC and Treg ${ }^{[8]}$.

However, a cancer patient's immune system is affected by other factors, such as depression and impairment of physical activity. Depression due to a cancer diagnosis, as well as chronic stress and social isolation, suppresses immune function by the adrenergic and glucocorticoid pathways ${ }^{[9]}$. The effects are especially strong on the lymphocytic stromal $\mathrm{TME}^{[10]}$, and are determinants in response to therapy.

Regarding physical activity, which could modify the depressive state, there are no conclusive data on the real impact on the immune system. Finally, although there is experience that correlates the amplitude of physical activity with the reduction of the risk of cancer-related and cardiovascular mortality ${ }^{[11]}$, this has not been reported on PDAC ${ }^{[12]}$.

The aim of this report is to analyze the characteristics of this long-term PDAC patient and the possible positive influence of long and constant physical activity on the global control of the disease.

\section{CASE REPORT}

A male 40-year-old patient has been recognized to have a locally advanced neoplasm of the pancreas head. After the appearance of obstructive jaundice, he was subjected to endoscopic retrograde cholangiopancreatography and positioning of biliary stenting with resolution of jaundice. Subsequently, he was subjected to exploratory laparotomy, which showed a locally advanced head pancreatic tumor that was judged inoperable. The intraoperative biopsy provided the diagnosis of PDAC. From December 2013 to July 2014, the patient underwent chemotherapy with Nab-PCT-GEM combination [Figure 1A and B] for seven cycles with radiological stability (not shown). Taking into consideration the persistence of non-operability, he performed RT $50 \mathrm{~Gy} / 15$ fractions combined to biweekly GEM with stabilization of the disease [Figure 1C and D].

After subsequent local progression, he was treated from April to October 2015 with FOLFIRINOX for six months with stabilization of the disease (not shown). This treatment resulted in bone marrow and peripheral neurological toxicity with negative repercussions on general conditions. After a slow and progressive clinical improvement, from February 2017, the patient undertook a constant course of physical exercise until he reached $15,000 \mathrm{~km}$ of walking. The reported daily route, carried out exclusively on foot, was on average $20 \mathrm{~km}$ and the total distance traveled was performed in 750 days.

During the five-year follow-up period, the stability status of the disease was confirmed [Figure $1 \mathrm{E}$ and F]. Furthermore, blood samples were taken the morning following a walk, periodically about every three months. The blood data are reported corresponding to the $\mathrm{km}$ traveled. 

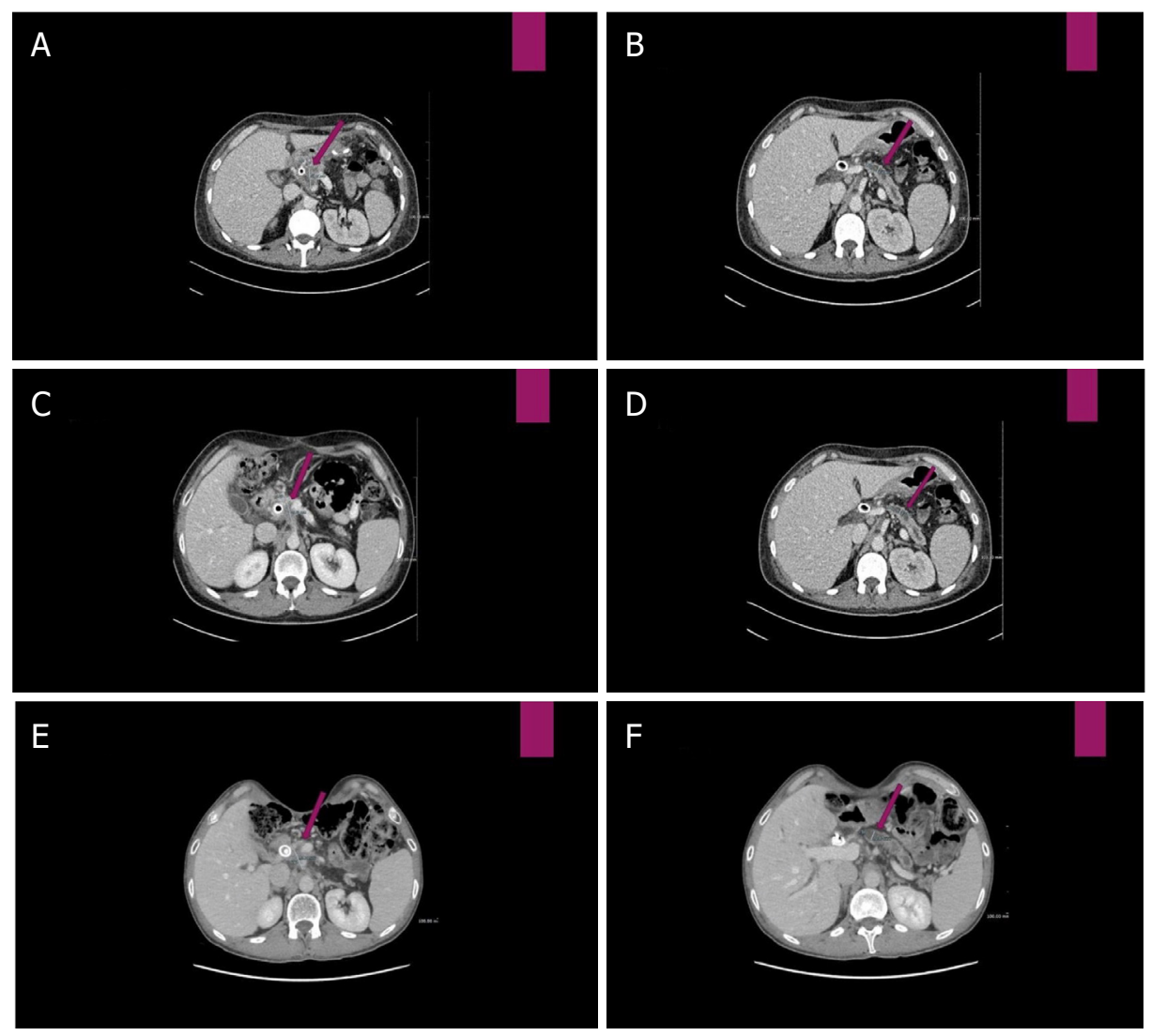

Figure 1. A: Computed tomography (CT) scan before Nab-paclitaxel-Gemcitabine therapy: hypodense lesion of 30 mm maximum transverse diameter, infiltrating the posterior lamina and presenting contact with the superior mesenteric artery; B: Wirsung duct dilatation of $8.42 \mathrm{~mm}$; C: CT scan after Nab-paclitaxel-Gemcitabine therapy and consolidation radiotherapy: volumetric stability of the pancreatic lesion $(29.76 \mathrm{~mm})$; D: the Wirsung duct caliber $(7.99 \mathrm{~mm}$ ); E: 63 months from start therapy (after Nab-paclitaxelGemcitabine, radiotherapy and FOLFIRINOX salvage therapy) presence of slight reduction of the pancreatic lesion (25.38 mm maximum transverse diameter); and F: increase of the Wirsung caliber $(10.67 \mathrm{~mm})$ with atrophy of the pancreas body and tail

The hematological study has documented a count reduction of lymphocytes and platelets during chemoradiotherapy followed by stabilization [Figure 2A]. Regarding the immune profile of lymphocyte subpopulations, a progressive reduction of $\mathrm{CD} 3, \mathrm{CD} 4$, and $\mathrm{CD} 8$ followed by an increase; a wave trend of CD16; and stability of HLA-DR and Treg were reported.

Moreover, after initial reduction of platelet/lymphocyte ratio followed by stabilization during therapy, an initial temporary increase tending to a fair final reduction after start of physical exercise was reported [Figure $2 \mathrm{~B}$ and $\mathrm{C}$ ]. Furthermore, after a decrease of Ca19.9 [Figure 2E] and C-reactive protein (CRP) and increase of albumin [Figure 2D], the stability over time of its level, as well as those of the other indexes of illness such as albumin [Figure 2D], CEA, and Ca19.9 [Figure 2E], was observed.

\section{DISCUSSION}

Current treatments for advanced PDAC represented by Nab-PCT-GEM and FOLFIRINOX combinations have improved prognosis and survival, which remain poor.

In addition to the aforementioned factors of a local nature, other factors of an immunological nature contribute to resistance to cytoreductive drugs and ultimately to neoplastic progression. 
A

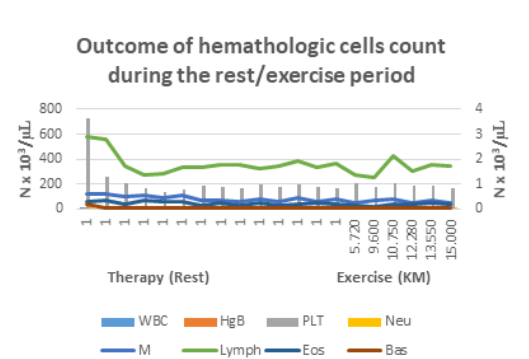

D

Outcome of CRP and albumine values during the rest/activity period

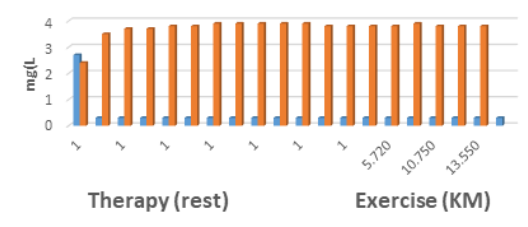

n CRP In albumine
B

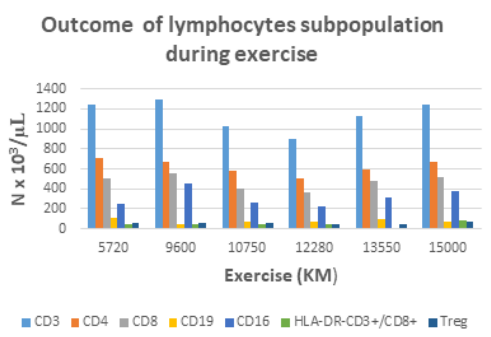

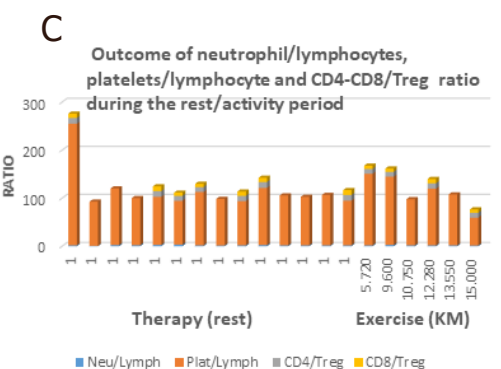

$\mathrm{E}$

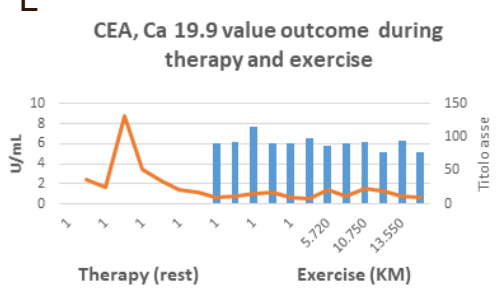

-

Figure 2. A: Hematologic outcome of population cells: decrease of platelets and lymphocytes count after therapy followed by stability of count cells during physical activity. Stability over time of hemoglobin level and white blood cells types; B: lymphocyte subpopulation during physical activity: progressive reduction of CD3, CD4 and CD8 followed by an increase, a wave trend of CD16 and stability of HLADR and Treg cells; C: initial reduction of Platelet/lymphocyte ratio followed by stabilization during therapy; it is followed by initial slight increase followed by stability and therefore slight reduction during exercise. Furthermore is shown a relative stability of neutrophil/ lymphocyte, CD4/Treg, CD8/Treg ratios; D: recovery of albumin levels and decrease of CRP after therapy followed by stability during physical activity; E: decrease of Ca 19.9 after therapy followed by the stability over time together of CEA levels during exercise

It is known how the magnitude of the tumor mutation burden correlates with the probability of response to the new checkpoint inhibitory agents that have recently entered clinical practice in the treatment of tumors.

PDAC is also negatively influenced by the low tumor mutation burden, characterized by low prevalence of somatic mutations and of neo-antigens expression with consequent resistance to new immunotherapeutic agents, such as anti-PD ${ }^{[13]}$. One of the key points of immunosuppression also present in PDAC is represented by the CXCR4/CXCL12 axis. In fact, from its block derives the promotion of intratumoral lymphocyte infiltration and the synergism of action with anti-PD-1 agent ${ }^{[14]}$.

Among the external factors, physical activity is very important in the homeostasis of the organism. It exercises antitumor activity in animal models and high levels of post-diagnosis exercise reduce the risk of recurrence and cancer-specific mortality in common solid tumors ${ }^{[15]}$. Such exercise must be undertaken gradually until a constant level is reached and maintained (inverted-J hypothesis), whereby regular moderate exercise improves immune system function while acute, intermittent efforts correlate with immunosuppression and increased susceptibility to cancer ${ }^{[16]}$.

Regarding the repercussions of physical activity and immune surveillance, the cells that are most stimulated are the natural killer (NK) cells, influenced by neuroendocrine ${ }^{[17,18]}$ and immunological mechanism such as IL-6 secretion resulting from muscle stress ${ }^{[19]}$.

However, discordant results have been reported in animal and clinical studies and were reiterated in a systematic literature review on the repercussions of physical exercise on NK cell counts and cytotoxicity. Indeed, definitive conclusions cannot yet be drawn because of the diversity in the intensity and duration of the exercise and in the time of blood sampling ${ }^{[20]}$. 
The emerging difficulties derive from the possible hormonal role on the total NK count, which does not include the tissue one, and from the correlations with performance status, age, and disease status in acute and chronic exercise $\mathrm{e}^{[20]}$.

Regarding innate immunity, immunological differences between healthy persons and cancer patients have been demonstrated ${ }^{[21,22]}$. In healthy persons, exercise can positively influence the pro-inflammatory polarization of M1 macrophages following immune escape ${ }^{[21]}$, and neutrophil counts ${ }^{[22]}$, while, in a tumor animal model, exercise caused vascular normalization and hypoxia reduction with improvement of response to chemotherapy ${ }^{[23]}$ and reduction of TAMs and neutrophils infiltrating their tumors determining tumor volume reduction ${ }^{[24]}$.

In the adaptive immune response, Th1-polarized $\mathrm{T}$ cells and Tregs, influenced by adhesion molecules, are involved in maintaining peripheral tolerance and appear to have a prognostic role ${ }^{[25-27]}$. Moderateintensity exercise produced in healthy 65 -year-old subjects a greater increase in lymphocyte response to immunogenic stimulation than in those who performed flexibility and toning exercises ${ }^{[28]}$. In fact, a single bout of physical exercise also seems to determine in the healthy an increase of differentiated NK-cells, $\mathrm{CD} 8+$, and $\gamma \delta$ T-cells, with marked sensitivity to external stimulation. This could have an implication in hematopoietic stem cell transplantation and represent a valid aid in the conventional treatment of tumors ${ }^{[29]}$.

Reduction of leukocyte count, improvement of depressive symptoms, and increase in diurnal salivary cortisol rhythm can be obtained in patients with a six-month exercise program differently from in sedentary patients but without the lymphocyte functional finding ${ }^{[18]}$.

Finally, in low immunogenic tumors, such as pancreatic adenocarcinoma, chemotherapy with drugs such as anthracyclines can trigger an immune response through dendritic cells (DC ${ }^{[30]}$.

Similar to chemotherapy, marathon running increases myeloid DC counts and the production of proand anti-inflammatory cytokines ${ }^{[31]}$. This finding supports the role of immunomodulatory mechanisms in the response and adaptation to acute, excessive exercise. The possible clinical application of these results has found a clinical finding in pretreated, unresponsive patients and in progression of disease, obtaining a disease control rate of $41 \%$, improvement of physical status, reduced risk of progression, and impact on increase of cytotoxic CD8+ CD28+ and decrease of suppressive CD8+ CD28- cell $^{[32]}$.

Regarding long-term survivors, the increased risk of developing second cancers and cardiovascular complications is known.

In this case, it also seems that the amplitude of physical activity reduced the risk of both cancer-related mortality and cardiovascular disease ${ }^{[11]}$. However, this was not found in PDAC ${ }^{[12]}$.

Nevertheless, there is some evidence for correlations among antiblastic treatment, moderate but constant physical exercise, and immune response in the neoplastic patients, although knowledge about the impact of physical activity on the immune system is not conclusive.

Cell death by chemotherapy might not be the single modality of antitumor action; there may be other immunomodulation mechanisms, regulated and controlled by immunosuppressive cells, that can condition the therapeutic response. They could act as an additive to chemotherapy and could improve the therapeutic index if "physical conditioning exercise" has been administered in selected patients before starting chemotherapy or new innovative therapy with checkpoint inhibitors. 
Therefore, if physical activity were recognized as a determining factor in the induction and maintenance of antitumor immune function, it could be used as adjuvant and maintenance therapies in the on and off phases of the active antineoplastic therapy, respectively.

Physical activity may have surprising positive results, especially in patients with a life-threatening disease, such as PDAC, who have undergone systemic treatments that cause long-term sequelae such as neurotoxicity with a negative impact on their quality of life. After a slow and progressive clinical improvement, our patient undertook a constant course of physical exercise until he reached more than $15,000 \mathrm{~km}$ of walking. These results are described in his book ${ }^{[33]}$, which deals with his manifold experiences, feelings, and personal reflections, as well as the clinical approach to his neoplastic disease. During the five-year follow-up period and walking activity, the stability of the disease was confirmed. Therefore, this could be linked to the contribution of the physical exercise as maintenance of response obtained after FOLFIRINOX. It is also worth noting the stability of the hematological and immunological profile, with a reduction trend of PLT/Lymph ratio, the normalization of CRP after treatment, and its maintenance over time during physical activity, which are indirect indices of immune surveillance. However, no statistical considerations can be made due to the lack of comparative data during an actual rest phase for the relative constant physical activity of the patient.

His unique experience should make us profoundly reflect on the effects of reactive depression to a cancer diagnosis, and to further study the relationships between physical activity and both treatment efficacy and immunosuppression. Prospective studies involving medical oncologists, immunologists, biologists, neurologists, and physiotherapists are needed to confirm these results.

\section{DECLARATIONS}

\section{Authors' contributions}

Conceptualization, data curation, formal analysis, investigation, methodology, project administration, software, supervision, validation, visualization, writing - original draft, writing - review \& editing, and used in attributes: Lo Re G, Magnaldi S, Doretto P, Innocente R, Lo Re F

\section{Availability of data and materials}

We declare that the patient's data derive from personal treatment and clinical-instrumental follow-up. The scientific data reported derive from the international literature and it is listed in the references.

\section{Financial support and sponsorship}

None.

\section{Conflicts of interest}

All authors declared that there are no conflicts of interest.

\section{Ethical approval and consent to participate}

Not applicable.

\section{Consent for publication}

Not applicable.

\section{Copyright}

(c) The Author(s) 2019. 


\section{REFERENCES}

1. Zhu Y, Herndon JM, Sojka DK, Kim KW, Knolhoff BL, et al. Tissue-resident macrophages in pancreatic ductal adenocarcinoma originate from embryonic hematopoiesis and promote tumor progression. Immunity 2017;47:323-38.

2. Mantovani A, Sozzani S, Locati M, Allavena P, Sica A. Macrophage polarization: tumor-associated macrophages as a paradigm for polarized M2 mononuclear phagocytes. Trends Immunol 2002;23:549-55.

3. Strauss L, Bergmann C, Szczepanski M, Gooding W, Johnson JT, et al. A unique subset of CD4+CD25highFoxp3+ T cells secreting IL10 and TGF- $\beta 1$ mediates suppression in the tumor microenvironment. Clin Cancer Res 2007;13:4345-54.

4. Gabrilovich DI, Nagaraj S. Myeloid-derived suppressor cells as regulators of the immune system. Nat Rev Immunol 2009;9:162-74.

5. Von Hoff DD, Ervin T, Arena FP, Chiorean EG, Infante J, et al. Increased survival in pancreatic cancer with nabpaclitaxel plus gemcitabine. N Engl J Med 2013;369:1691-703.

6. Conroy T, Desseigne F, Ychou M, Bouché O, Guimbaud R, et al. FOLFIRINOX versus gemcitabine for metastatic pancreatic cancer. New Engl J Med 2011;364:1817-25.

7. Cullis J, Siolas D, Avanzi A, Barui S, Maitra A, et al. Macropinocytosis of nabpaclitaxel drives macrophage activation in pancreatic cancer. Cancer Immunol Res 2017;5:182-90.

8. Shevchenko I, Karakhanova S, Soltek S, Link J, Bayry J, et al. Low-dose gemcitabine depletes regulatory T cells and improves survival in the orthotopic Panc02 model of pancreatic cancer. Int J Cancer 2013;133:98-107.

9. Reiche EM, Nunes SO, Morimoto HK. Stress, depression, the immune system, and cancer. Lancet Oncol 2004;5:617-25.

10. Lutgendorf SK, Lamkin DM, DeGeest K, Anderson B, Dao M, et al. Depressed and anxious mood and T-cell cytokine producing populations in ovarian cancer patients. Brain Behav Immun 2008;22:890-900.

11. Lee IM, Wolin KY, Freeman SE, Sattlemair J, Sesso HD. Physical activity and survival after cancer diagnosis in men. J Phys Act Health 2014;11:85-90.

12. Lee IM, Sesso HD, Oguma Y, Paffenbarger RS Jr. Physical activity, body weight, and pancreatic cancer mortality. Br J Cancer 2003;88:679-83.

13. Brahmer JR, Tykodi SS, Chow LQ, Hwu WJ, Topalian SL, et al. Safety and activity of anti-PD-L1 antibody in patients with advanced cancer. N Engl J Med 2012;366:2455-65.

14. Hidalgo M, Epelbaum R, Wolpin BM, Stemmer M, Geva R, et al. A Phase 2a Trial to Assess the Safety and Efficacy of BL-8040 and Pembrolizumab in Patients with Metastatic Pancreatic Adenocarcinoma (PDAC). Ann Oncol 2018;29: viii400-41.

15. Ballard-Barbash R, Friedenreich CM, Courneya KS, Siddiqi SM, McTiernan A, et al. Physical activity, biomarkers, and disease outcomes in cancer survivors: a systematic review. J Natl Cancer Inst 2012;104:815-40.

16. Woods JA, Davis JM, Smith JA, Nieman DC. Exercise and cellular innate immune function. Med Sci Sports Exerc 1999;31:57-66.

17. Zheng X, Cui XX, Huang MT, Liu Y, Wagner GC, et al. Inhibition of progression of androgen-dependent prostate LNCaP tumors to androgen independence in SCID mice by oral caffeine and voluntary exercise. Nutr Cancer 2012; 64:1029-37.

18. Saxton JM, Scott EJ, Daley AJ, Woodroofe M, Mutrie N, et al. Effects of an exercise and hypocaloric healthy eating intervention on indices of psychological health status, hypothalamic-pituitary adrenal axis regulation and immune function after early-stage breast cancer: a randomised controlled trial. Breast Cancer Res 2014;16:R39.

19. Ellingsgaard H, Hojman P, Pedersen BK. Exercise and health - emerging roles of IL-6. Curr Opin Physiol 2019;10:49-54.

20. Zimmer P, Schenk A, Kieven M, Holthaus M, Lehmann J, et al. Exercise induced alterations in NK-cell cytotoxicity - methodological issues and future perspectives. Exerc Immunol Rev 2017;23:66-81.

21. Koelwyn GJ, Wennerberg E, Demaria S, Jones LW. Exercise in regulation of inflammation-immune axis function in cancer initiation and progression. Oncology (Williston Park) 2015;29:908-20.

22. Peake JM. Exercise-induced alterations in neutrophil degranulation and respiratory burst activity: possible mechanisms of action. Exerc Immunol Rev 2002;8:49-100.

23. Betof AS, Lascola CD, Weitzel D, Landon C1, Scarbrough PM, et al. Modulation of murine breast tumor vascularity, hypoxia and chemotherapeutic response by exercise. J Natl Cancer Inst 2015;107:djv040.

24. Almeida PW, Gomes-Filho A, Ferreira AJ, Rodrigues CE, Dias-Peixoto MF, et al. Swim training suppresses tumor growth in mice. J Appl Physiol (1985) 2009;107:261-5.

25. Galon J, Costes A, Sanchez-Cabo F, Kirilovsky A, Mlecnik B, et al. Type, density, and location of immune cells within human colorectal tumors predict clinical outcome. Science 2006;313:1960-4.

26. Bindea G, Mlecnik B, Tosolini M, Kirilovsky A, Waldner M, et al. Spatiotemporal dynamics of intratumoral immune cells reveal the immune landscape in human cancer. Immunity 2013;39:782-95.

27. Facciabene A, Motz GT, Coukos. T-regulatory cells: key players in tumor immune escape and angiogenesis. Cancer Res 2012;72:216271.

28. Woods JA, Ceddia MA, Wolters BW, Evans JK, Lu Q, et al. Effects of 6 months of moderate aerobic exercise training on immune function in the elderly. Mech Ageing Dev 1999;109:1-19.

29. Simpson RJ, Bigley AB, Agha N, Hanley PJ, Bollard CM, et al. Mobilizing immune cells with exercise for cancer immunotherapy. Exerc Sport Sci Rev 2017;45:163-72.

30. Obeid M, Tesniere A, Ghiringhelli F, Fimia GM, Apetoh L, et al. Calreticulin exposure dictates the immunogenicity of cancer cell death Nat Med 2007;13:54-61.

31. Nickel T, Emslander I, Sisic Z, David R, Schmaderer C, et al. Modulation of dendritic cells and toll-like receptors by marathon running. Eur J Appl Physiol 2012;112:1699-708. 
32. Zhao YJ, Jiang N, Song QK, Wu JP, Song YG, et al. Continuous DC-CIK infusions restore CD8+ cellular immunity, physical activity and improve clinical efficacy in advanced cancer patients unresponsive to conventional treatments. Asian Pac J Cancer Prev 2015;16:2419-23.

33. Spinelli A. Se cammino, vivo. Se di cancro si muore, pur si vive. Available from: https://www.lafeltrinelli.it/libri/andrea-spinelli/secammino-vivo-se-cancro/9788865492680. [Last accessed on 13 Dec 2019] 